\title{
Na Induced Changes in the Electronic Band Structure of Graphene Grown on C-Face SiC
}

\author{
Leif I. Johansson, Chao Xia, Chariya Virojanadara \\ Department of Physics, Chemistry and Biology, Linköping University, Linköping, Sweden \\ Email: lejoh@ifm.liu.se
}

Received October 2, 2012; revised November 16, 2012; accepted December 28, 2012

\begin{abstract}
Studies of the effects induced on the electron band structure after Na deposition, and subsequent heating, on a C-face 2 MLs graphene sample are reported. Na deposition shifts the Dirac point downwards from the Fermi level by about 0.5 $\mathrm{eV}$ due to electron doping. After heating at temperatures from around $120^{\circ} \mathrm{C}$ to $300^{\circ} \mathrm{C}$, the $\pi$-band appears considerably broadened. Collected Si $2 \mathrm{p}$ and $\mathrm{Na} 2 \mathrm{p}$ spectra then indicate $\mathrm{Na}$ intercalation in between the graphene layers and at the graphene $\mathrm{SiC}$ interface. The broadening is therefore interpreted to arise from the presence of two slightly shifted, but not clearly resolved, $\pi$-bands. Constant energy photoelectron distribution patterns, $\mathrm{E}\left(\mathrm{k}_{\mathrm{x}}, \mathrm{k}_{\mathrm{y}}\right) ; \mathrm{s}$, extracted from the clean 2 MLs graphene $\mathrm{C}$-face sample look very similar to earlier calculated distribution patterns for monolayer, but not Bernal stacked bi-layer, graphene. After Na deposition the patterns extracted at energies below the Dirac point appear very similar so the doping had no pronounced effect on the shape or intensity distribution. At energies above the Dirac point the extracted angular distribution patterns show the flipped, "mirrored", intensity distribution predicted for monolayer graphene at these energies. An additional weaker outer band is also discernable at energies above the Dirac point, which presumably is induced by the deposited Na.
\end{abstract}

Keywords: Graphene on C-Face SiC; Graphene Band Structure; Na Intercalation; Constant Energy Photoelectron; Angular Distribution Patterns

\section{Introduction}

Graphene grown epitaxially on the wide band semiconductor $\mathrm{SiC}$ is considered a most promising platform [1] for future carbon-based electronic devices. Chemical approaches adapted from the field of graphite intercalation compounds [2] have been used for tailoring the electronic properties of graphene. For example, sodium $(\mathrm{Na})$ deposited on monolayer graphene grown on $\mathrm{Si}$-face $\mathrm{SiC}$ intercalates [3-5] first between the carbon layers but with time also underneath the carbon buffer layer. Heating at temperatures around $100^{\circ} \mathrm{C}$ promotes intercalation [3-5] but at higher temperatures $\mathrm{Na}$ starts to de-intercalate and desorb from the sample. The intercalation transforms the carbon buffer layer to a second graphene layer, as revealed by the appearance of a second $\pi$-band. The graphene layers become fairly strongly n-doped by the $\mathrm{Na}$, as revealed by a downward shift of the Dirac point by about $1.0 \mathrm{eV}$. Studies [6,7] of the electron band structure of graphene grown on $\mathrm{Si}$-face $\mathrm{SiC}$ show that the number of $\pi$-bands corresponds directly to the number of graphene layers. The effects induced by other alkali metals have also been investigated quite thoroughly and there is a general consensus about these findings [8-11].
For graphene grown on $\mathrm{C}$-face $\mathrm{SiC}$ the situation is quite different. Only one $\pi$-band ( $\pi$-cone) with the Dirac point located close to the Fermi level has in general been observed [12-14] even for multilayer graphene samples. For Bernal (AB) stacking of graphene layers a single $\pi$-band with linear dispersion at the $\bar{K}$-point results [15] only for single layer graphene (SLG), while bi- and trilayer graphene show $[15,16]$ two and three split parabolic $\pi$-bands at the $\bar{K}$-point, respectively. Calculations of how the misorientation of stacked graphene layers affects the electronic structure have demonstrated that the band structure of SLG is also found in incommensurate multilayered graphene systems. The macro Low Energy Electron Diffraction (LEED) pattern from C-face graphene is smeared out into a strongly modulated diffraction ring [12]. This, together with surface X-ray diffraction results [17] has been interpreted to indicate that the graphene layers on the C-face stack in such a way that adjacent layers are rotated with respect to each other. A recent investigation [14] of C-face graphene using Low Energy Electron Microscopy (LEEM), X-ray Photoelectron Electron Microscopy (XPEEM) and selected area (micro) LEED showed however unambiguously formation of fairly large crystallographic grains of multilayer graphene 
where there was no rotational disorder between adjacent layers but that the grains had different azimuthal orientations. The Angle Resolved Photo Electron Spectroscopy (ARPES) results [14] showed a single $\pi$-cone with the Dirac point located close to the Fermi level. Therefore further studies of the electron band structure of C-face graphene are well motivated.

In this study the effects induced in the electron band structure of C-face graphene are investigated after depositing similar amounts of $\mathrm{Na}$ and after heating at similar temperatures as earlier done [3,5] for Si-face graphene samples. The idea was to see if similar effects could be induced on $\mathrm{C}$-face graphene, that the Dirac point would shift downwards by one $\mathrm{eV}$ after deposition and if more than one $\pi$-band could be observed after $\mathrm{Na}$ deposition and heating. Since Na do intercalate Si-face graphene we assumed that it would also intercalate $\mathrm{C}$-face graphene that is known to form in smaller domains/grains and thus have a higher density of defects on the surface.

\section{Experimental}

A graphene sample grown [14] on a n-type $6 \mathrm{H}-\mathrm{SiC}$ substrate at $1800^{\circ} \mathrm{C}$ for $20 \mathrm{~min}$ in an Ar pressure of $500 \mathrm{mbar}$ was utilized. A graphene coverage of predominantly 2 MLs was determined using LEEM, Photo Electron Spectroscopy (PES) and LEED at beam line I311 at the MAX laboratory. This beam line is equipped with a modified SX-700 monochromator that provides light for two endstations. One for PES, built around a large hemispherical Scienta electron analyzer, providing a total energy resolution of 10 to $100 \mathrm{meV}$ at photon energies from 45 to $450 \mathrm{eV}$. The other is built up around a LEEM (LEEM III, Elmitec $\mathrm{GmbH}$ ) instrument. These stations were utilized for characterizing the graphene sample. ARPES experiments were performed at beam line I4, which is equipped with a SGM monochromator and a PHOIBOS 100 2D CCD Specs energy analyzer. The low angular dispersion (LAD) lens mode was selected, providing an acceptant angle of $\pm 7^{\circ}$. A Ta foil mounted on the sample manipulator was utilized as a reference sample for determining the Fermi level. Deposition of Na was performed as earlier $[3,5]$ using a SAES getter source and with the sample at room temperature. Subsequent heating was carried out for five minutes at different selected temperatures. The sample temperature was determined using optical pyrometers in the temperature range $350^{\circ} \mathrm{C}-950^{\circ} \mathrm{C}$. Lower sample temperatures had to be estimated from extrapolation of the input power (current $\times$ voltage) and sample temperature relation determined over the temperature range covered by the pyrometers. The uncertainty in the low temperatures range is therefore large, ca. $\pm 25^{\circ} \mathrm{C}$. Data collection was carried out with the sample at room temperature.

\section{Results and Discussion}

A LEEM image collected at a voltage of $3.3 \mathrm{eV}$ and a field of view of $10 \mathrm{~mm}$ is shown in Figure 1(a). The number of graphene layers present in the different domains is determined from the electron reflectivity versus electron kinetic energy, i.e. the so called I-V curve. The reflectivity curves extracted from the areas labeled from $A$ to $P$ are shown in Figure 1(b). Curves A to I are very similar and show two pronounced minima that indicate a coverage of 2 MLs. Curves $\mathrm{J}$ to $\mathrm{N}$ also show two minima indicating a coverage of $2 \mathrm{MLs}$, although these curves have a slightly different shape around $2 \mathrm{eV}$. Curve $\mathrm{O}$ show one dip indicating single graphene layer coverage and curve $\mathrm{P}$ represents an area with no graphene coverage. Most of the surface of this sample is thus found to have a graphene coverage of 2 MLs. Selected area LEED diffraction patterns collected from areas $\mathrm{E}$ and $\mathrm{D}$ at an energy of $45 \mathrm{eV}$ are shown in Figure 1(c). A probing area with a diameter of $0.4 \mu \mathrm{m}$ was used so both patterns are collected from a single grain/domain, but the two domains are seen to have different azimuthal orientations. No rotational disorder between the two graphene layers is however discernable in these diffraction patterns.

C 1s spectra collected from this 2 MLs graphene sample at three photon energies are shown in Figure 2(a). They are modeled accurately using only two components, the $\mathrm{SiC}$ component from the substrate and the $\mathrm{G}$ com-
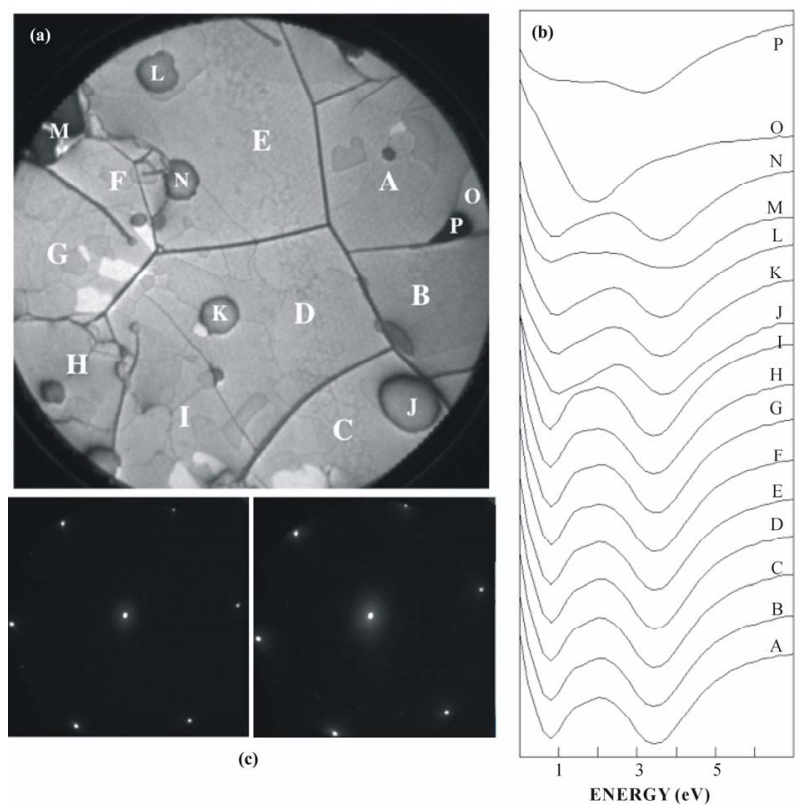

Figure 1. (a) LEEM image recorded at $0.4 \mathrm{eV}$ and a field of view of $10 \mu \mathrm{m}$; (b) Electron reflectivity curves extracted from the different areas labeled $A$ to $P$ in (a), showing a coverage of mainly 2 ML of grapheme; (c) Selected area LEED patterns, collected at $45 \mathrm{eV}$ using a probing area diameter of $0.4 \mu \mathrm{m}$, from two different grains/domains in (a). 

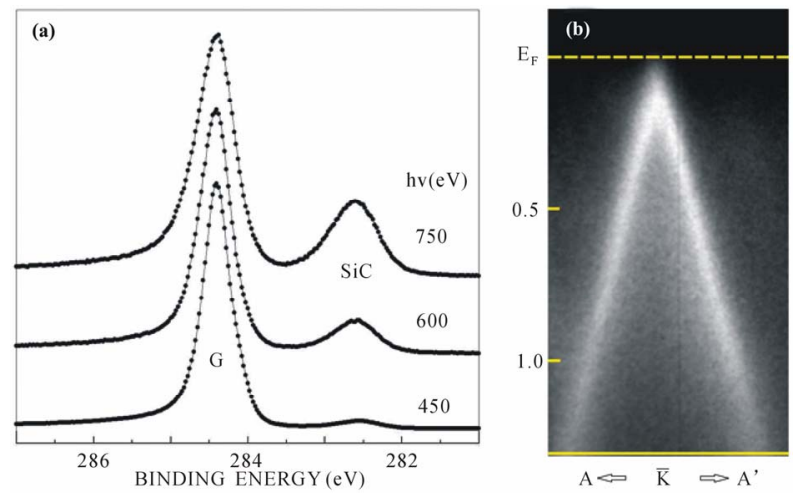

Figure 2. (a) $\mathrm{C}$ 1s spectrum recorded using three different photon energies showing contribution from graphene (G) and the $\mathrm{SiC}$ substrate; (b) The $\pi$-band recorded around the $\bar{K}$-point at a photon energy of $33 \mathrm{eV}$.

ponent from the graphene layers. In agreement with earlier results $[14,18]$ no trace of a carbon buffer layer component can be observed. The energy separation of $1.9 \mathrm{eV}$ and peak intensity ratio of around 6 (at $600 \mathrm{eV}$ ), determined between the graphene and the SiC components $(\mathrm{G} / \mathrm{SiC})$ are consistent with previously determined values [18] for a graphene thickness of about 2 MLs. The $\pi$-band recorded from this 2 MLs graphene sample around the $\bar{K}$ point and along the A- $\bar{K}-\mathrm{A}^{\prime}$ direction using a photon energy of $33 \mathrm{eV}$ is shown in Figure 2(b). The Dirac point is located within ca. $75 \mathrm{meV}$ below the Fermi level indicating a slight n-doping instead of the slight p-doping earlier reported [13]. Since the graphene grain size was only on the order of a few micrometers, a few $\pi$-cones from domains of different azimuthal orientations were typically detected when moving the light spot over the sample. However, the Dirac point of the different cones appeared always located at the same energy position. After $\mathrm{Na}$ deposition and subsequent heating the sample was always relocated to the same position where only one $\pi$-cone appeared in the spectrum from the clean surface.

The effects induced in the $\pi$-band structure close to the $\bar{K}$ point after $\mathrm{Na}$ deposition and subsequent heating are shown in Figure 3. The Dirac point is seen to shift down by about $0.5 \mathrm{eV}$ after $\mathrm{Na}$ deposition. On $\mathrm{Si}$-face graphene the downward shift [3] was twice as large, i.e. ca. $1.0 \mathrm{eV}$ for a similar amount of deposited $\mathrm{Na}$. Subsequent heating at $50^{\circ} \mathrm{C}$ and $80^{\circ} \mathrm{C}$ is seen to only make the band appear slightly more diffuse. Heating at temperatures from $120^{\circ} \mathrm{C}$ to $300^{\circ} \mathrm{C}$ results in a considerable broadening of the spectral features. At $400^{\circ} \mathrm{C}$ the Dirac point has moved back quite much towards $\mathrm{E}_{\mathrm{F}}$ so most of the $\mathrm{Na}$ appears to have desorbed, and at $950^{\circ} \mathrm{C}$ the initial band is restored. An interesting thing is what causes the broader features that are observed particularly well at $200^{\circ} \mathrm{C}$ and $300^{\circ} \mathrm{C}$. First we speculate based on earlier findings, and then we present supporting experimental evidence. On Si-face graphene intercalation of $\mathrm{Na}$ in between the carbon layers and also at the graphene $\mathrm{SiC}$ interface was strongly promoted [3,5] by heating at around $100^{\circ} \mathrm{C}$ while at higher temperatures the $\mathrm{Na}$ started to de-intercalate and desorb from the surface, Therefore we suggest similar things $[3,5]$ to occur also on $\mathrm{C}$-face graphene. After deposition and after heating at temperatures below ca. $100^{\circ} \mathrm{C}$ a large part of the $\mathrm{Na}$ stays on the surface and in between the graphene layers. When heating at temperatures from $120^{\circ} \mathrm{C}$ to $300^{\circ} \mathrm{C}$ intercalation at the interface and in between the carbon layers is promoted, while the $\mathrm{Na}$ on the surface decreases considerably since Na begins to desorb. Then the two carbon layers experience a different electron $n$-type doping level so two $\pi$-bands, cones, are present, one with the Dirac point fairly close to $E_{F}$ and the other with the Dirac point at around $0.4 \mathrm{eV}$ below $\mathrm{E}_{\mathrm{F}}$. After heating at $400^{\circ} \mathrm{C}$ most of the $\mathrm{Na}$ has desorbed but there is still some intercalated $\mathrm{Na}$, giving rise to a smaller energy difference between the Dirac points of the two graphene layers. Intercalation at the interface can be unambiguously identified by the appearance $[3,5]$ of shifted substrate components in the C 1s and Si $2 p$ core level spectra. However, on beam line $\mathrm{I} 4$ the $\mathrm{C} 1 \mathrm{~s}$ level is not accessible but a limited set of $\mathrm{Si} 2 \mathrm{p}$ and $\mathrm{Na}$ $2 p$ spectra were possible to collect, see Figure 4, and they provide support for the above speculations.

The Si $2 p$ spectra, displayed in the left panel in Figure 4, show that the Si $2 p$ signal is strongly attenuated after deposition of $\mathrm{Na}$ and that some intercalation may eventually have occurred. After heating at $120^{\circ} \mathrm{C}$ and $200^{\circ} \mathrm{C}$ a Si $2 p$ component shifted about two eV to lower binding energy is clearly revealed, which indicates intercalation $[3,5]$ at the graphene $\mathrm{SiC}$ interface. At $400^{\circ} \mathrm{C}$ this component is not discernable so most of the $\mathrm{Na}$ then appears to have left the interface. The $\mathrm{Na} 2 \mathrm{p}$ spectra displayed in the right panel tell a similar story. After deposition a main sharp component, corresponding to Na metal on top of the graphene [3,5], and a broad feature at higher binding energy are observed. After heating at $120^{\circ} \mathrm{C}$ only two broad components are observed, like earlier [3,5] for $\mathrm{Na}$ deposited on $\mathrm{Si}$ face graphene. The new component at lower binding energy was then identified to correspond to $\mathrm{Na}$ intercalated at the graphene $\mathrm{SiC}$ interface and the one at higher binding energy to $\mathrm{Na}$ intercalated in between the graphene layers. When increasing the heating temperature to $200^{\circ} \mathrm{C}$ and then to $400^{\circ} \mathrm{C}$ only the component corresponding to intercalation in between the graphene layers remains, but at $950^{\circ} \mathrm{C}$ also this component vanished. Thus the evolution of the core level spectra support the speculations concerning the reason for the changes induced in the electron band structure after heating at different temperatures.

Since the Dirac point is shifted downwards from the Fermi level after $\mathrm{Na}$ deposition it is then possible to in- 

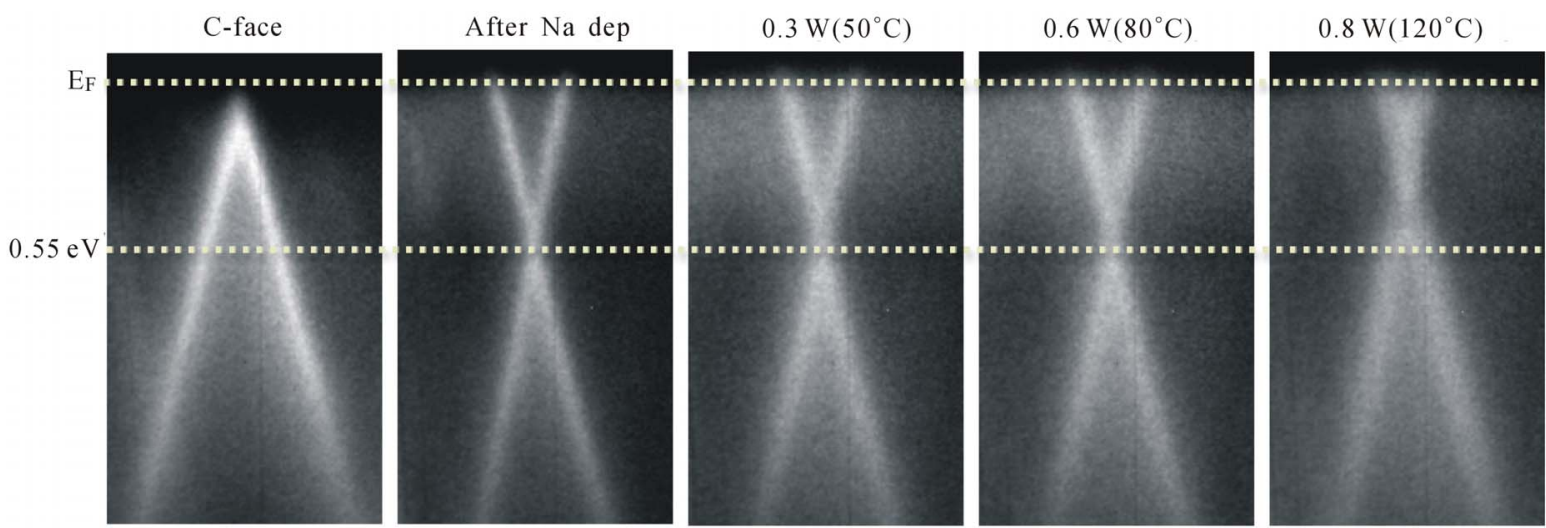

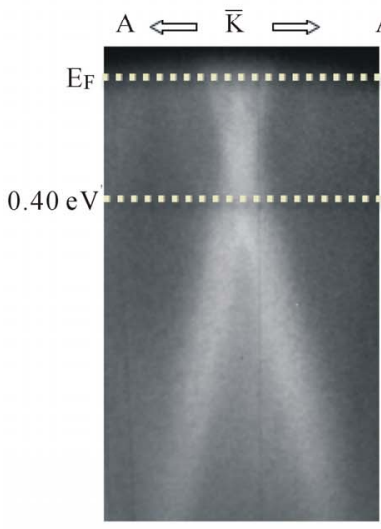

$0.8 \mathrm{~W}\left(120^{\circ} \mathrm{C}\right)$

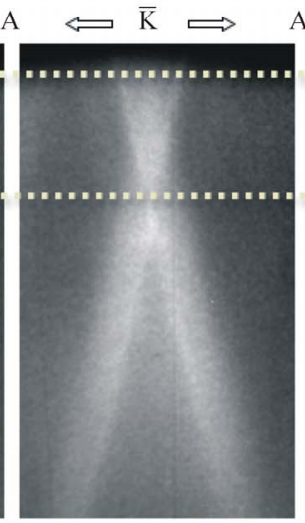

$1.5 \mathrm{~W}\left(200^{\circ} \mathrm{C}\right)$

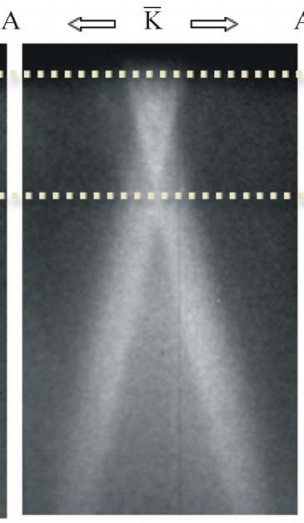

$3 \mathrm{~W}\left(300^{\circ} \mathrm{C}\right)$

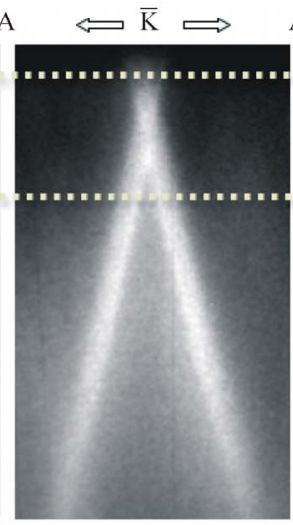

$5 \mathrm{~W}\left(400^{\circ} \mathrm{C}\right)$

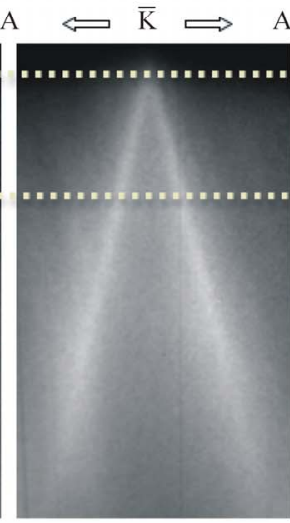

$950^{\circ} \mathrm{C}$

Figure 3. The $\pi$-band structure recorded close to the $\bar{K}$ point before and after Na deposition and after heating at different temperatures. A photon energy of $33 \mathrm{eV}$ was used.

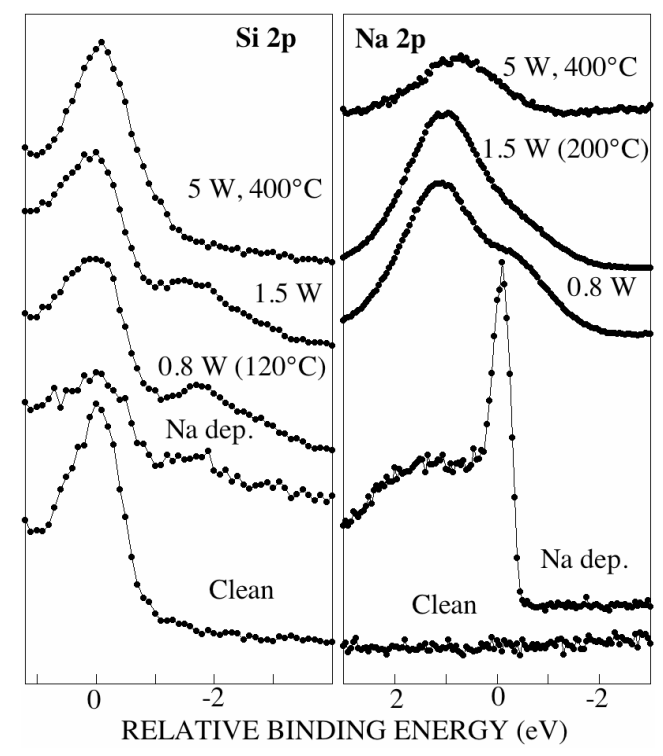

Figure 4. Normal emission Si $2 p$ and Na $2 p$ spectra recorded before and after $\mathrm{Na}$ deposition and after heating at three different temperatures. A photon energy of $140 \mathrm{eV}$ was used.

vestigate the band structure both above and below the Dirac point and compare that with the band structure from the clean surface and with calculated results. For that purpose $\mathrm{E}\left(\mathrm{k}_{\mathrm{x}}, \mathrm{k}_{\mathrm{y}}\right)$ angular distribution patterns were extracted around the $\bar{K}$-point at certain energies relative to the Dirac point. For the clean 2 MLs graphene $\mathrm{C}$-face sample this pattern is a distinct small circular spot at $E_{D}$, see upper panel in Figure 5 in which $E\left(k_{x}, k_{y}\right)$; $s$ from the clean surface are displayed. When moving away from the Dirac point the pattern has at $0.2 \mathrm{eV}$ the shape of a circular ring which when moving further away becomes gradually more triangular. The intensity distribution and shape of these constant energy maps at and below the Dirac point agree well with the patterns calculated [19] for monolayer, but not bi-layer, graphene. Close to the Dirac point the calculated pattern for monolayer graphene is fairly circular and when moving further away it becomes more triangular due to the increased effect of triangular warping, see Figure 2 in [19]. The asymmetry in the intensity distribution, the appearance of the so called dark corridor [19,20], has for monolayer graphene been well accounted for by considering the two source interference pattern from the two inequivalent atomic sites in the graphene honeycomb lattice. After $\mathrm{Na}$ deposition the patterns extracted below $\mathrm{E}_{\mathrm{D}}$ appear fairly similar to those for the clean surface, see lower panel 


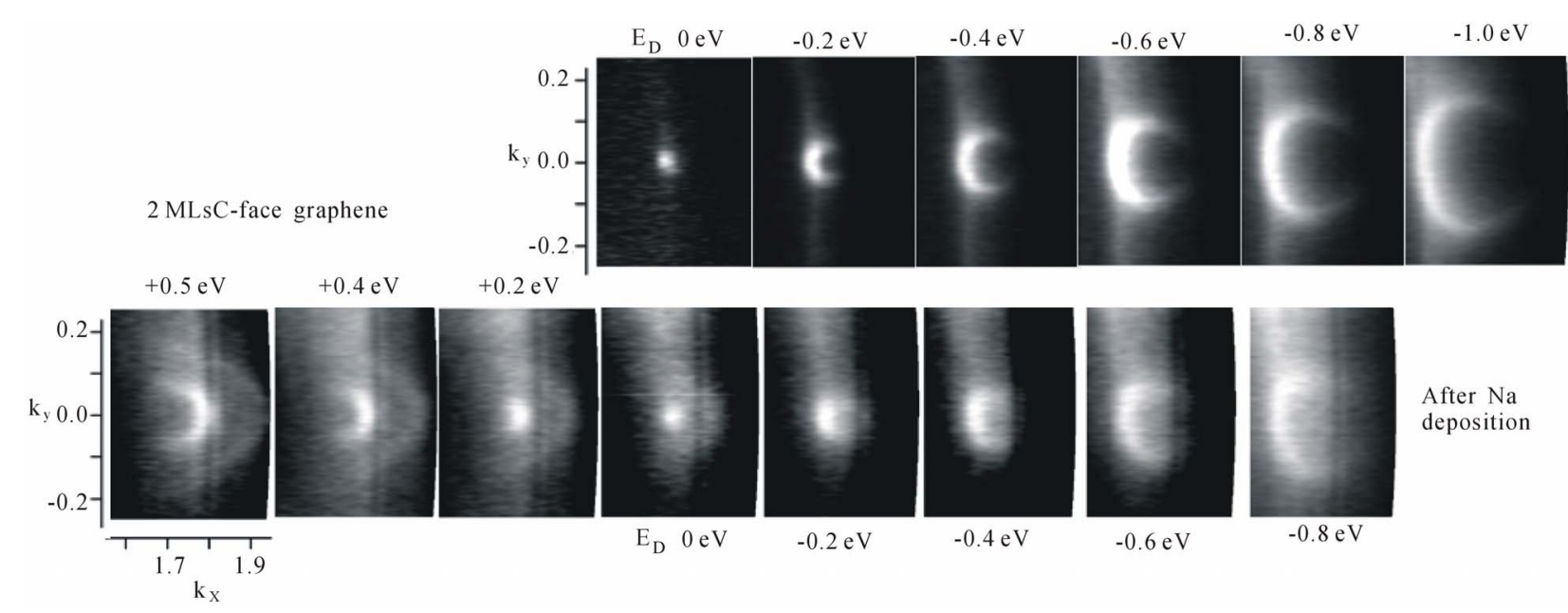

Figure 5. Photoelectron angular distribution patterns $\mathbf{E}\left(\mathbf{k}_{\mathrm{x}}, \mathbf{k}_{\mathrm{y}}\right)$ around the $\bar{K}$-point extracted from recorded ARPES spectra collected from the graphene sample before (upper panel) and after (lower panel) Na deposition. The wavevector $k_{\mathrm{x}}$ is along the $\bar{\Gamma}-\bar{K}-\bar{M}$ direction and $\mathbf{k}_{\mathbf{y}}$ along $\mathbf{A}-\bar{K}-\mathrm{A}^{\prime}$.

in Figure 5. However, the circular spot at $E_{D}$ is distinctly larger and the patterns are slightly more diffuse but the doping has not induced any other visible changes in the symmetry of the pattern. When looking at the patterns extracted above $\mathrm{E}_{\mathrm{D}}$ they are flipped, "mirrored", compared to the pattern at the same energy below $E_{D}$. This was also predicted [19] at energies located above $E_{D}$ for monolayer graphene, see Figure 2 in [19], but not for Bernal stacked bi-layer graphene. Thus both for the as grown 2 MLs graphene C-face sample and for the doped graphene after $\mathrm{Na}$ deposition the $\mathrm{E}\left(\mathrm{k}_{\mathrm{x}}, \mathrm{k}_{\mathrm{y}}\right)$ angular distribution patterns extracted reflect those predicted for monolayer graphene, while the patterns predicted for Bernal stacked bilayer graphene look distinctly different, especially at energies below the Dirac point, see Figure 4 in [19]. It also deserves to be noticed that the $\pi$-band dispersion we observe for $\mathrm{Na}$ doped 2 MLs graphene close to the $\bar{K}$ point looks distinctly different compared to that in Figures 3(c) and (d) in [13], where a hatlike shape of the bi-layer bands was reported. It is thus clear that the two graphene layers on our C-face sample are not Bernal stacked. The micro LEED patterns in Figure 1(c) moreover show that the layers are not misoriented, i.e. rotated relative to each other, so the stacking is neither turbostratic. Therefore AA stacking seems the only available option left $[15,16]$ for multilayer graphene showing linear dispersion at the $\bar{K}$ point, since Bernal (AB) and ABC stacking results in "hatlike" parabolic dispersions and split bands at the $\bar{K}$ point. However, a splitting of the $\pi$-bands from different layers is also predicted for AA stacking, but this has not yet been possible to reveal or verify experimentally. Therefore we speculate that the interaction and charge transfer between graphene layers grown on $\mathrm{C}$-face $\mathrm{SiC}$ is maybe so weak/small that the band splitting is not resolvable in conventional ARPES.
At energies above $\mathrm{E}_{\mathrm{D}}$ there is after $\mathrm{Na}$ deposition also an additional weaker "outer band" discernable in the patterns, in Figure 5, which monolayer graphene cannot explain the presence of. To further elucidate its presence cuts from the collected data set, are shown in Figure 6. In cut a), made at the $\bar{K}$ point, i.e. at $\mathrm{k}_{\mathrm{x}} \approx 1.7[1 / \AA \AA]$, and along the A- $\bar{K}-\mathrm{A}^{\prime}$ direction, this additional band is barely visible. In cut b), at the $\bar{K}$ point but along the $\bar{\Gamma}-\bar{K}-\bar{M}$ direction, it appears as a weak feature on the right hand side of the main $\pi$-band. In cut c), however, made at $\mathrm{k}_{\mathrm{x}} \approx 1.8[1 / \AA \AA]$ and along the $\mathrm{A}-\bar{K}$-A' direction, this additional band becomes more visible. We can only point out the presence of this weak band, induced by the $\mathrm{Na}$ deposited on the 2 MLs graphene sample, but have no further explanation for it.

\section{Summary and Conclusions}

Deposition of $\mathrm{Na}$ on a $2 \mathrm{MLs}$ graphene $\mathrm{C}$-face sample shifted the Dirac point downwards from the Fermi level by about $0.5 \mathrm{eV}$ due to electron n-type doping. Subsequent heating at temperatures from ca. $120^{\circ} \mathrm{C}$ to $300^{\circ} \mathrm{C}$ broadened the $\pi$-band considerably. Collected Si $2 p$ and $\mathrm{Na} 2 \mathrm{p}$ spectra then indicated $\mathrm{Na}$ intercalation in between the graphene layers and at the graphene $\mathrm{SiC}$ interface. The broadening is therefore suggested to arise from presence of two $\pi$-bands, slightly shifted to each other by a difference in electron doping concentration of the graphene layers.

Constant energy photoelectron distribution patterns, E $\left(\mathrm{k}_{\mathrm{x}}, \mathrm{k}_{\mathrm{y}}\right)$; $\mathrm{s}$, extracted for the clean 2 MLs graphene C-face sample look very similar to earlier calculated distribution patterns for monolayer graphene, but not the patterns for Bernal stacked bi-layer graphene. After $\mathrm{Na}$ deposition the $\mathrm{E}\left(\mathrm{k}_{\mathrm{x}}, \mathrm{k}_{\mathrm{y}}\right)$ patterns extracted at energies below the 


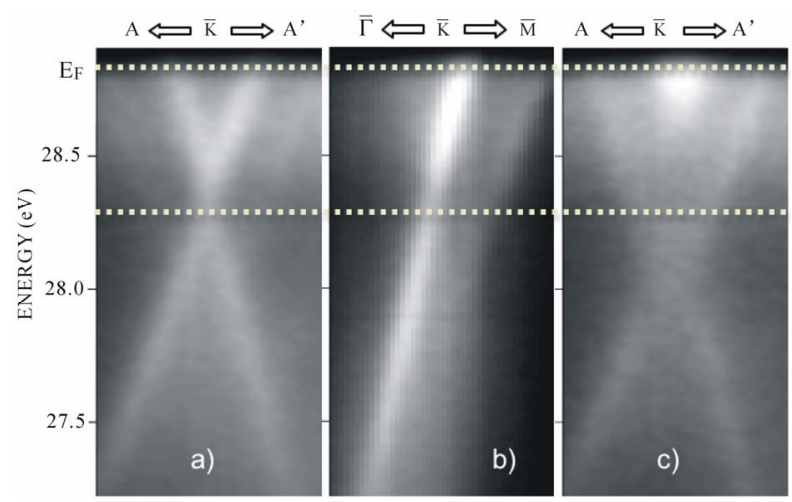

Figure 6. Cuts from the data set recorded after Na deposition in Figure 5. In a) the cut is along the $A-\bar{K}-A^{\prime}$ direction

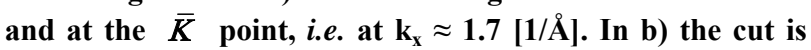
also at the $\bar{K}$ point but along the $\bar{\Gamma}-\bar{K}-\bar{M}$ direction. In c) the cut is along the $A-\bar{K}-A^{\prime}$, direction but at $k_{x} \approx 1.8$ [1/Å], see lower panel in Figure 5.

Dirac point appear very similar to those for the clean sample so the doping did not visibly affect the shape or intensity distribution. Then at energies above the Dirac point the angular distribution patterns show the flipped, "mirrored", intensity distribution predicted for monolayer, but not bi-layer, graphene. The deposited $\mathrm{Na}$ induced an additional weaker outer band at energies above the Dirac point, which calculated results for monolayer graphene could not account for.

\section{Acknowledgements}

The authors gratefully acknowledge support from the European Science Foundation, within the EuroGRAPHENE (EPIGRAT) program, and the Swedish Research Council (\#621-2011-4252 and Linnaeus Grant).

\section{REFERENCES}

[1] P. N. First, W. A. de Heer, T. Seyller, C. Berger, J. A. Stroscio and J.-S. Moon, "Epitaxial Graphenes on Silicon Carbide," MRS Bulletin, Vol. 35, No. 4, 2010, pp. 296305. doi: $10.1557 / \mathrm{mrs} 2010.552$

[2] M. S. Dresselhaus and G. Dresselhaus, "Intercalation Compounds of Graphite," Advances in Physics, Vol. 51, No. 1, 2002, pp. 1-186. doi:10.1080/00018730110113644

[3] S. Watcharinyanon, L. I. Johansson, C. Xia and C. Virojanadara, "Changes in Structural and Electronic Properties of Graphene Grown on $6 \mathrm{H}-\mathrm{SiC}(0001)$ Induced by $\mathrm{Na}$ Deposition," Journal of Applied Physics, Vol. 111, No. 8, 2012, Article ID: 083711. doi:10.1063/1.4704396

[4] A. Sandin, T. Jayasekera, J. E. Rowe, K. W. Kim, M. B. Nardelli and D. B. Dougherty, "Multiple Coexisting Intercalation Structures of Sodium in Epitaxial GrapheneSiC Interfaces," Physical Review B, Vol. 85, 2012, Article ID: 125410 . doi:10.1103/PhysRevB.85.125410

[5] C. Xia, S. Watcharinyanon, A. A. Zakharov, L. I. Johans- son, R. Yakimova and C. Virojanadara, "Detailed Studies of $\mathrm{Na}$ Intercalation on Furnace-Grown Graphene on $6 \mathrm{H}$ $\mathrm{SiC}(0001)$, , in Press.

[6] T. Ohta, A. Bostwick, J. L. Mc-Chesney, T. Seyller, K. Horn and E. Rotenberg, "Interlayer Interaction and Electronic Screening in Multilayer Graphene Investigated with Angle-Resolved Photoemission Spectroscopy," Physical Review Letters, Vol. 98, No. 20, 2007, Article ID: 206802. doi:10.1103/PhysRevLett.98.206802

[7] A. Bostwick, T. Ohta, T. Seyller, K. Horn and E. Rotenberg, "Quasiparticle Dynamics in Graphen," Nature Physics, Vol. 3, 2007, pp. 36-40. doi:10.1038/nphys477

[8] A. A. Bostwick, F. Speck, T. Seyller, K. Horn, M. Polini, R. Asgari, A. H. MacDonald and E. Rotenberg, "Observation of Plasmarons in Quasi-Freestanding Doped Graphene," Science, Vol. 328, No. 5981, 2010, pp. 999-1002. doi:10.1126/science.1186489

[9] C. Virojanadara, S. Watcharinyanon, A. A. Zakharov and L. I. Johansson, "Epitaxial Graphene on $6 \mathrm{H}$-SiC and $\mathrm{Li}$ Intercalation," Physical Review B, Vol. 82, No. 20, 2010, Article ID: 205402. doi:10.1103/PhysRevB.82.205402

[10] S. Watcharinyanon, C. Virojanadara and L. I. Johansson, "Rb and Cs Deposition on Epitaxial Graphene Grown on 6H-SiC(0001)," Surface Science, Vol. 605, No. 21-22, 2011, pp. 1918-1922. doi:10.1016/j.susc.2011.07.007

[11] A. L. Walter, A. Bostwick, K.-J. Jeon, F. Speck, M. Ostler, T. Seyller, L. Moreschini, Y. J. Chang, M. Polini, R. Asgari, A. H. MacDonald, K. Horn and E. Rotenberg, "Effective Screening and the Plasmaron Bands in Graphene," Physical Review B, Vol. 84, No. 8, 2011, Article ID: 085410. doi:10.1103/PhysRevB.84.085410

[12] M. Sprinkle, D. Siegel, Y. Hu, J. Hicks, A. Tejeda, A. Taleb-Ibrahimi, P. Le Fe'vre, F. Bertran, S. Vizzini, H. Enriquez, S. Chiang, P. Soukiassian, C. Berger, W. A. de Heer, A. Lanzara and E. H. Conrad, "First Direct Observation of a Nearly Ideal Graphene Band Structure," Physical Review Letters, Vol. 103, No. 22, 2009, Article ID: 226803. doi:10.1103/PhysRevLett.103.226803

[13] D. A. Siegel, C. G. Hwang, A. W. Fedorov and A. Lanzara, "Quasifreestanding Multilayer Graphene Films on the Carbon Face of SiC," Physical Review B, Vol. 81, No. 24, 2010, Article ID: 241417. doi:10.1103/PhysRevB.81.241417

[14] L. I. Johansson, S. Watcharinyanon, A. A. Zakharov, T. Iakimo, R. Yakimova and C. Virojanadara, "Stacking of Adjacent Graphene Layers Grown on C-Face SiC," Physical Review B, Vol. 84, No. 12, 2011, Article ID: 125405. doi:10.1103/PhysRevB.84.125405

[15] S. Latil, V. Meunier and L. Henrard, "Massless Fermions in Multilayer Graphitic Systems with Misoriented Layers: $A b$ Initio Calculations and Experimental Fingerprints," Physical Review B, Vol. 76, No. 20, 2007, Article ID: 201402. doi:10.1103/PhysRevB.76.201402

[16] S. Latil and L. Henrard, "Charge Carriers in Few-Layer Graphene Films," Physical Review Letters, Vol. 97, No. 3, 2006, Article ID: 036803. doi:10.1103/PhysRevLett.97.036803

[17] J. Hass, R. Feng, J. E. Millan-Otoya, X. Li, M. Sprinkle, 
P. N. First, W. A. de Heer, E. H. Conrad and C. Berger, "Structural Properties of the Multilayer Graphene/4H-Si (000-1) System as Determined by Surface X-Ray Diffraction," Physical Review B, Vol. 75, No. 21, 2007, Article ID: 214109. doi:10.1103/PhysRevB.75.214109

[18] K. V. Emtsev, F. Speck, Th. Seyller, L. Ley and J. D. Riley, "Interaction, Growth, and Ordering of Epitaxial Graphene on SiC $\{0001\}$ Surfaces: A Comparative Photoelectron Spectroscopy Study," Physical Review B, Vol. 77, No. 15, 2008, Article ID: 155303. doi:10.1103/PhysRevB.77.155303

[19] M. Mucha-Kruczyński, O. Tsyplyatyev, A. Grishin, E.
McCann, V. I. Fal'ko, A. Bostwick and E. Rotenberg, "Characterization of Graphene through Anisotropy of Constant-Energy Maps in Angle-Resolved Photoemission," Physical Review B, Vol. 77, No. 19, 2008, Article ID: 195403. doi:10.1103/PhysRevB.77.195403

[20] I. Gierz, J. Henk, H. Höchst, C. R. Ast and K. Kern, "Illuminating the Dark Corridor in Graphene: Polarization Dependence of Angle-Resolved Photoemission Spectroscopy on Graphene," Physical Review B, Vol. 83, No. 12, 2011, Article ID: 121408. doi:10.1103/PhysRevB.83.121408 